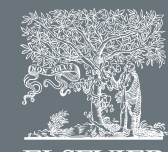

ELSEVIER

\title{
IT Future of Medicine: from molecular analysis to clinical diagnosis and improved treatment
}

\section{Valeria Zazzu ${ }^{1,3}$, Babette Regierer ${ }^{1,3}$, Alexander Kühn², Ralf Sudbrak ${ }^{1,3}$ and Hans Lehrach ${ }^{1,3}$}

\footnotetext{
${ }^{1}$ Max Planck Institute for Molecular Genetics, Ihnestrasse 63-73, 14195 Berlin, Germany

2 Alacris Theranostics GmbH, Fabeckstraße 60-62, 14195 Berlin, Germany
}

The IT Future of Medicine (ITFoM, http://www.itfom.eu/) initiative will produce computational models of individuals to enable the prediction of their future health risks, progression of diseases and selection and efficacy of treatments while minimising side effects. To be able to move our health care system to treat patients as individuals rather than as members of larger, divergent groups, the ITFoM initiative, proposes to integrate molecular, physiological and anatomical data of every person in 'virtual patient' models. The establishment of such 'virtual patient' models is now possible due to the enormous progress in analytical techniques, particularly in the '-omics' technology areas and in imaging, as well as in sensor technologies, but also due to the immense developments in the ICT field.

As one of six Future and Emerging Technologies (FET) Flagship Pilot Projects funded by the European Commission, ITFoM with more than 150 academic and industrial partners from 34 countries, will foster the development in functional genomics and computer technologies to generate 'virtual patient' models to make them available for clinical application. The increase in the capacity of next generation sequencing systems will enable the high-throughput analysis of a large number of individuals generating huge amounts of genome, epigenome and transcriptome data, but making it feasible to apply deep sequencing in the clinic to characterise not only the patient's genome, but also individual samples, for example, from tumours. The genome profile will be integrated with proteome and metabolome information generated via new powerful chromatography, mass spectrometry and nuclear magnetic resonance techniques. The individualised model will not only enable the analysis of the current situation, but will allow the prediction of the response of the patient to different therapy options or intolerance for certain drugs. 


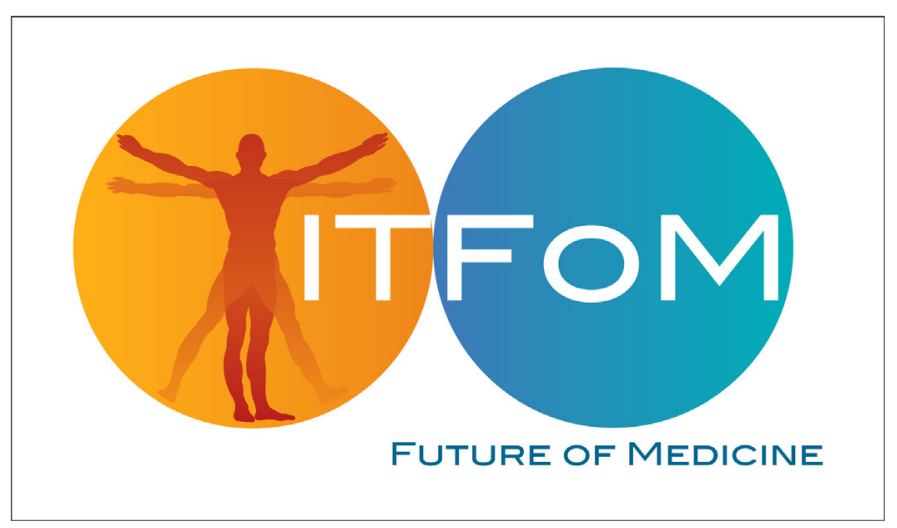

\section{From molecular analysis to clinical diagnostic}

The global initiative to sequence the human genome with the publication of the first result was a fundamental breakthrough in $2001[1,2]$ and had an enormous influence on the molecular biology and the entire field of life sciences.

The molecular analysis, in particular the '-omics' technologies, have been developed subsequently over the last 10 years to grasp the totality of molecules present in organelles, cells, tissues and organs in order to obtain an overall view of a biological system. The development of the '-omics' technologies aims, besides the optimisation of precision, throughput, multiplexing, speed and miniaturisation, also at the reduction in the cost per sample, to allow a routine use in the hospital.

The sequencing technologies take the lead in this development; very recently market leaders in sequencing technology announced the launching of new products able to analyse a human genome within one day at a price of 1.000 USD. Especially in cancer, the tumour tissue sequencing will be the central basis for treatment decisions. Since we know now that not just any patient, but also every tumour is different, sequencing technologies will help to detect which mutations cause the tumour to grow. Based on this information, in the future a doctor will be much more accurate in choosing the right treatment for the individual patient.

With increasing knowledge about the functionality of living systems, our understanding will continue to grow about protein modifications and interactions as well as the structure of proteins. A highly dynamic development can be seen also in the imaging technologies that give a more precise idea of what is going on in the body of a patient. The integration of molecular imaging technologies and sensors will allow us to get an insight view about molecule behaviour in space and time.

These innovations in analytical technologies in molecular biology and their application in medicine induced a paradigm shift allowing for the application of a new way for medical diagnosis and treatment. With appropriate diagnostics the clinician or general practitioner can tailor a medical treatment or a prevention strategy to an individual patient according to the responding markers on the gene, protein or metabolite level.

\section{Systems medicine - from clinical diagnosis to improved treatment}

From pharmacogenomics studies it became clear that the common drug blockbuster strategy ('one drug fits all') must be revised according to the individual responses [3]. It could be shown for instance that there is a very high number (70-100\%) of nonresponders for various cancer drugs indicating that for most cancer patients a treatment will only generate severe side-effects without leverage for the disease itself [4].

Thanks to systems biology, a new discipline within the life sciences, the model-based approaches in human life science are so promising that they are expected to induce the next paradigm shift in modern medicine towards an individual-centred health care. Based on the molecular methods, systems medicine breaks boundaries deciphering the complex mechanisms of diseases, accelerates the discovery of new treatments and supports the evaluation of clinical trials; systems medicine can also be used to design new molecular diagnostics tools.

Systems biology, with methods and tools for mathematical analysis integrating and interpreting biological data, develops and builds mathematical models that allow a simulation and visualisation of biological processes. Cellular interaction networks, which are central to the understanding of biological systems, can be well represented with the help of mathematical model approaches [5-7]. The mathematical models developed in systems biology support the integration and analysis of increasingly large data sets and combine them with existing knowledge to enable an intelligent interpretation of the data.

Especially in cancer and tumour development, the signalling pathways play a central role in a mathematical model; groundbreaking for a basic understanding of the components of cancer signalling pathways was the publication by Hanahan and Weinberg in 2000 [8] with an update in 2011 [9]. The methods of systems biology developed systematically for more than ten years require further extensive development to describe adequately the highly complex processes of a living system (cell, tissue or organ) and their interrelation. Other challenges are to combine and integrate the heterogeneous data in the model, and this applies for the integration of genomic data as well as for data from other levels of biological information such as proteins and metabolites.

The PyBios model, which has been developed at the Max Planck Institute for Molecular Genetics, focuses on signal transduction pathways in cancer [8,9]; this model is using Monte Carlo simulations to study the interaction between the components and to identify mutations with influence on the system [10].

More information levels will be considered to be integrated in the future, for instance the metagenome of a human body since new publications show an influence of intestinal bacteria on the health status $[11,12]$. The metagenomic (sequencing) analysis of the intestinal bacterial population of a human ('microbiome') will provide a powerful analytical method that might be integrated in the future as a standard procedure in clinical diagnostics.

So far it was still too difficult to capture and integrate most information about lifestyle and environmental data for the patient diagnosis. For the preparation of a comprehensive and unique image of a patient, however, this additional information will be very important to ensure adequate patient-tailored therapy but also to develop a robust prevention strategy.

\section{IT Future of Medicine - the future of personalised medicine}

New ground-breaking developments in diagnostic methods now allow a very precise detection of the health status of a 


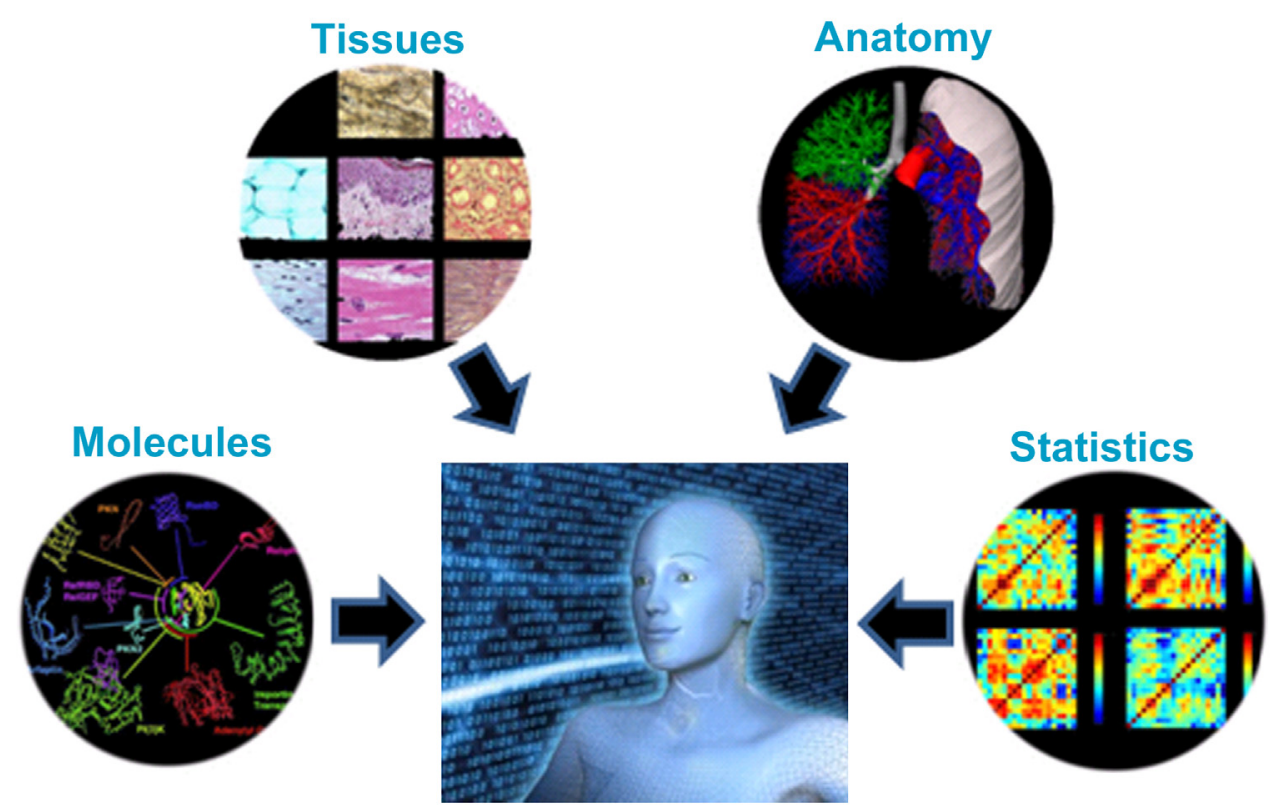

\section{FIG. 1}

The ITFoM computational models will integrate all of the types of all relevant genomic, epigenomic, functional information, imaging, pathology, environment and metagenomics data, but also individual patient histories as well as the individualised patient models derived from this information accessible to the consenting patient and his doctor. This comprehensive information package will be integrated with patient clinical records in the same way as blood chemistry, X-rays, MRI results, among others, are currently integrated.

patient and his disease. But how can this information for the individual patient be used efficiently? The European initiative 'IT Future of Medicine' is answering this question through the development of a new, data-rich computational model based on integrated molecular, physiological and anatomical data of every person, the 'virtual patient', and the identification of new avenues for treatment and prevention approaches (Fig. 1).

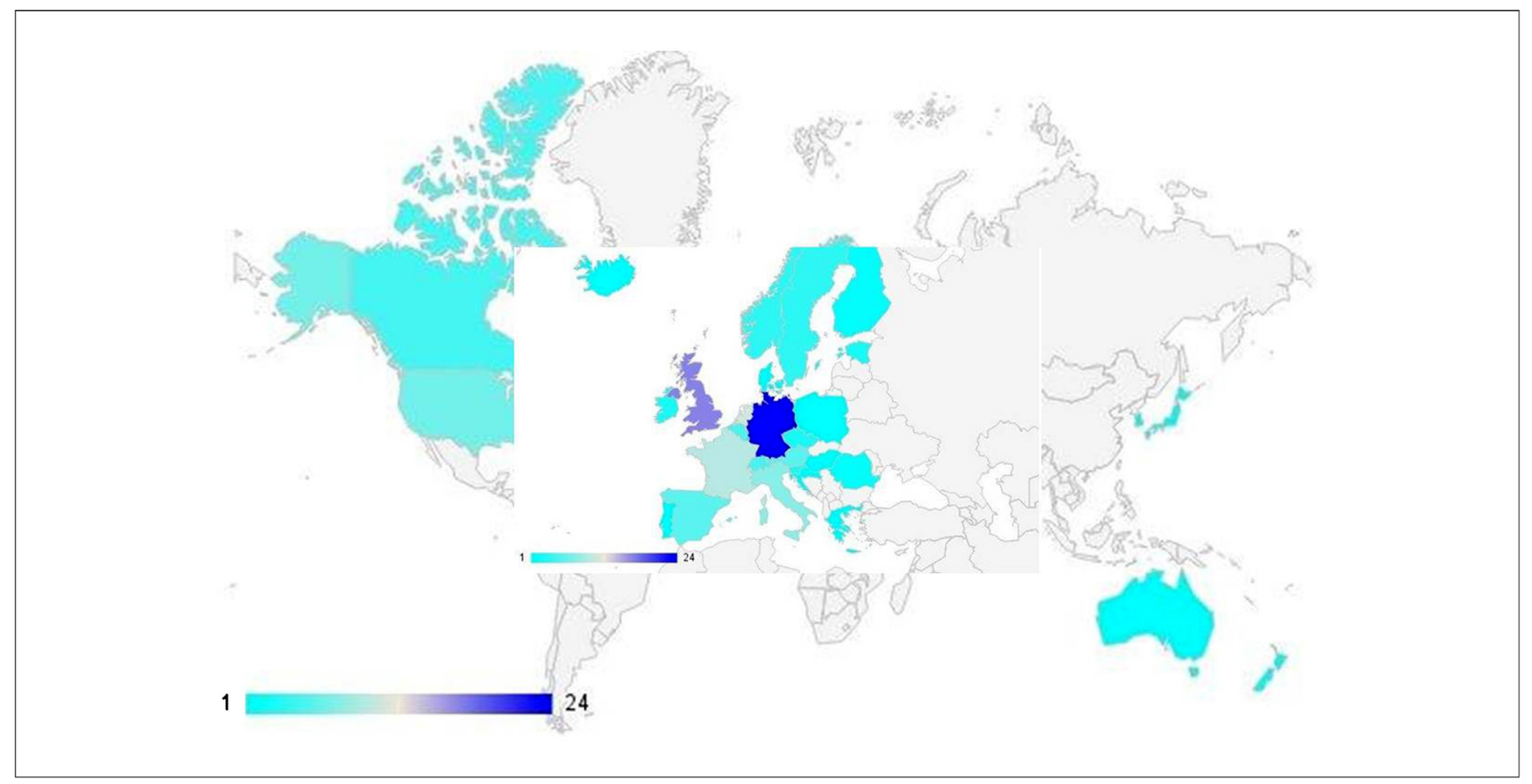

FIG. 2

The map shows the location of partnering institutions in ITFoM. The colour intensity indicates the number of ITFoM partners in each country. 
To cope with the enormous amount of data that will soon be generated in modern medicine, targeted Information and Communication Technology (ICT) developments will be a prerequisite to empower the 'virtual patient' model for the clinical practice. Solutions for topics like data processing, automation, storage, compression and in-time availability are highly relevant to enable the 'virtual patient'. Efficient pipelines of samples and resulting data from patient to model have to be implemented. The transfer of the information from model to clinician is a critical point for the acceptance of the new system in the day-to-day practice, for example, visualisation and human-computer interfaces in particular are demanding issues that must be optimised for a successful implementation [13].

All these developments must be accompanied by measures assuring that legal and ethical issues connected to the 'virtual patient' are adequately addressed and integrated from the beginning. Therefore, the lively interaction with stakeholder groups and especially patient organisations are highly important for the success of the approach.

To develop a 'virtual patient' and to apply it for the clinic routine, further refinement of the analytical technologies is necessary, but in parallel also the development of solutions for the computing requirements to process and handle the large amounts of heterogeneous and complex data. In the ITFoM consortium, experts from the medical practice, the analytical technology development and the ICT have joined from all over the world in order to face this big challenge in a global approach (Fig. 2).

\section{Acknowledgements}

The authors wish to thank the various research organisations that support the work that has led up to the ITFoM concept. The ITFoM pilot initiative was funded by Grant 285609, FP7.

\section{References}

[1] International Human Genome Sequencing Consortium. Nature 2001;409:860 921.

[2] Venter JC, Adams MD, Myers EW, Li PW, Mural RJ, Sutton GG, et al. Science 2001;291(5507):1304-51.

[3] Kalow W. The Pharmacogenomics Journal 2006;6:162-5.

[4] Park DJ, Stoehlmacher J, Lenz HJ. Current Opinion in Pharmacology 2003;3(4):378-85

[5] Noble, D. The music of life: Biology beyond genes, Oxford University Press, p. $176 ; 2006$.

[6] Bader S, Kühner S, Gavin AC. FEBS Letters 2008;582(8):1220-4.

[7] Klipp E. FEBS Letters 2009;583(24):4013-8.

[8] Hanahan D, Weinberg RA. Cell 2000;100(1):57-70.

[9] Hanahan D, Weinberg RA. Cell 2011;144(5):646-74.

[10] Wierling C, Kühn A, Hache H, Daskalaki A, Maschke-Dutz E, Peycheva S. Mutation Research 2012;746(2):163-70.

[11] Turnbaugh PJ, Hamady M, Yatsunenko T, Cantarel BL, Duncan A, Ley RE. Nature 2009;457(7228):480-4.

[12] Arumugam M, Raes J, Pelletier E, Le Paslier D, Yamada T, Mende DR. Nature 2011;473(7346):174-80.

[13] Lehrach H, Subrak R, Boyle P, Pasterk M, Zatloukal K, Muller H. Procedia Computer Science 2011;7:26-9.

\section{Links}

Web

http://www.itfom.eu/

http://www.fet-f.eu/

http://www.itfomthemovie.eu/

https://www.facebook.com/itfom

http://www.linkedin.com/groups/IT-Future-Medicine

Twitter: \#ITFoM

Email: itfom@molgen.mpg.de 